\title{
Simulations of L-band Staring Radar Moving Target Integration Efficiency
}

\author{
Fabiola Gersone and Alessio Balleri \\ Centre for Electronic Warfare, Information and Cyber \\ Cranfield University, Defence Academy of the UK \\ Shrivenham, SN6 8LA, UK \\ a.balleri@cranfield.ac.uk
}

\author{
Chris J Baker \\ School of Engineering \\ University of Birminghan \\ Birminghan, B15 2TT, UK \\ C.J.Baker.1@bham.ac.uk
}

\author{
Mohammed Jahangir \\ Aveillant Ltd \\ Cambridge, CB24 9AD, UK \\ mohammed.jahangir@aveillant.com
}

\begin{abstract}
Aveillant Ltd has developed a staring L-band radar that deploys a static quasi-monostatic antenna in transmission and a static digital phased array on receive capable of generating multiple simultaneous beams. Because the antenna is not rotating, the radar can stare at targets and select long dwell times with no effect on the scan rate. High Doppler resolution can be achieved and used to detect small targets, such as drones, even in heavy clutter. Despite the staring array, targets moving with a variable radial velocity generate echoes with a timevarying Doppler frequency shift that limits the integration gain achievable with standard Fourier Transform based techniques. As a result, the number of pulses can be integrated remains limited to the effective coherent processing interval with a consequent suboptimal Signal to Noise Ratio (SNR).

This paper presents the results of a set of simulations aimed at studying the integration gain efficiency of a staring radar of the type of the Aveillant Holographic radar for targets moving with a constant and non-constant radial velocity. The case of a target flying horizontally with respect to the radar boresight is investigated to show that compensation techniques can be potentially employed to maximise coherence on the target and the resulting integration gain.
\end{abstract}

\section{INTRODUCTION}

Traditional scanning radars use a rotating antenna that combines a transmitter and a receiver. As they rotate, transmit and receive they are able to capture returns from objects for only a limited period of time. The total time the radar spends on a target (time on target or dwell time) is the result of a trade-off between the desired Doppler resolution and the total time to scan the scene under surveillance. To increase the Doppler resolution, that is the capability of the radar to resolve targets with different speeds, a longer dwell time on the target is required but, if the radar spends a longer time in every directions, the time it takes to scan all directions increases. The resulting compromise is that, on average, only approximately $1 \%$ of each scan is spent on a target and the radar cannot differentiate between different types of moving objects.

The 3D Holographic radar developed by Aveillant Ltd is a staring radar that uses a different solution. The radar deploys a static quasi monostatic transmitting antenna and a staring array on receive that can continuously receive echoes from targets in the radar beam (about $100 \%$ of the time). Element level digitalisation permits the provision of multiple simultaneous receive beams under design control and with a near continuous refresh rate. This configuration enables the sensor to achieve wide angular coverage without the need to rotate the antenna. The solution of using a separate wide-beam broadcasting transmitter allows $100 \%$ time on target and provides a realtime, three-dimensional surveillance of the airspace. The radar provides a significant amount of information on the object and so can create a signature that can be characterised and identified. As the sensor is able to continuously stare at the target, the Doppler resolution is very high and can be used to measure and exploit the target micro-Doppler signature characteristics [1]. For applications of Air Traffic Control (ATC), the continuous dwell on the target and high Doppler resolution are exploited to fine tune post detection filter processes that reject false alarms and preserve only detections from genuine aircraft [2].

Despite the very long illumination time provided by staring radars, standard Fourier based integration techniques rely on the assumption of a constant radial velocity component and hence a constant frequency Doppler. As a result, the coherent integration time is limited by the target trajectory characteristics, even when the target response remains correlated. Classical detection techniques require the use of a large bank of filters which may be difficult to implement in practice ( [3]) and hence research looking at how targets can be focused for improved detection is attracting significant interest.

This paper presents an analysis of the integration gain efficiency of traditional signal processing based on Fourier techniques for a staring radar. The results of a set of simulations with targets moving with a constant and non-constant radial velocity and for a Gaussian target response characterised by an autocorrelation function that decays exponentially with time. The model for a target flying horizontally with respect to the radar boresight is investigated to show that compensation techniques can be potentially developed and employed to maximise the resulting integration gain.

\section{THEORY}

Let us consider a transmitted staring radar signal of amplitude $A_{T}$ centred around a carrier frequency $f_{0}$ of the form

$$
s_{T}(t)=A_{T} \tilde{p}(t) e^{j 2 \pi f_{0} t}
$$


with $\tilde{p}(t)$ being the complex envelope of the transmitted signal of unit energy.

The echo produced by a moving target received by the $n$-th antenna element of the receiving phased array is an attenuated and delayed replica of the transmitted signal

$$
s_{n}(t)=\tilde{b} \tilde{p}\left(t-\tau_{n}(t)\right) e^{-j 2 \pi f_{0} \tau_{n}(t)} e^{j 2 \pi f_{0} t}
$$

where

$$
\tau_{n}(t)=\frac{R_{n}(t)}{c}=\frac{R_{r n(t)}}{c}+\frac{R_{t}(t)}{c}
$$

with $R_{r n(t)}$ and $R_{t}(t)$ being the distance between the target and the $n$-th receiving element and between the transmitter and the target, respectively.

A target moving along Line Of Sight (LOS) with constant radial velocity $V_{r}$ at a distance from the radar much greater than the antenna size can be described the dynamics equation

$$
R_{n}(t)=R_{n, 0}+2 V_{r} t
$$

where $R_{n, 0}=R_{r n}(0)+R_{t}(0)$ is the target bistatic range at $t=0$ relative to the receiving $n$-th element. The resulting echo time delay is

$$
\tau_{n}(t)=\frac{R_{n, 0}}{c}+\frac{2 V_{r}}{c} t=\tau_{n}(0)+\frac{2 V_{r}}{c} t
$$

After replacing Eq. 5 in Eq. 2 the target echo can be expressed as

$s_{n}(t)=\tilde{b} \tilde{p}\left(t-\tau_{n}(0)-\frac{2 V_{r}}{c} t\right) e^{-j 2 \pi f_{0} \tau_{n}(0)} e^{-j 2 \pi f_{0} \frac{2 V_{r}}{c}} e^{j 2 \pi f_{0} t}$

which after down-conversion becomes

$$
\tilde{s}_{n}(t)=\tilde{b} \tilde{p}\left(t-\tau_{n}(0)-\frac{2 V_{r}}{c} t\right) e^{-j 2 \pi f_{0} \tau_{n}(0)} e^{j 2 \pi f_{D} t}
$$

where $f_{d}=-f_{0} \frac{2 V_{r}}{c}$ is the Doppler frequency shift induced by the target.

Narrow band signals satisfy the inequality $\frac{2 V_{r} T}{c} \ll \frac{1}{B}$ and, as a consequence, the time compression of the complex envelope can be neglected to obtain [3]

$$
\tilde{s}_{n}(t)=\tilde{b} \tilde{p}\left(t-\tau_{n}(0)\right) e^{-j 2 \pi f_{0} \tau_{n}(0)} e^{j 2 \pi f_{D} t}
$$

Finally, the total echo signal after beamforming with an array consisting of $N \times M$ elements with weight $w_{n}$ is

$$
\tilde{s}_{R}(t)=\sum_{n=1}^{N \times M} w_{n} \tilde{s}_{n}(t)
$$

For a pulse radar with Pulse Repetition Interval (PRI) $T$, the complex envelope of the transmitted signal can be written as

$$
\tilde{p}(t)=\sum_{i=0}^{N_{p}-1} \tilde{f}(t-i T)
$$

and Fourier based techniques, such as range-Doppler maps, are used to compensate the Doppler term of the received signal and provide optimal coherent integration.
When the target is moving with a variable radial velocity $v(t)$, the target dynamics are such that

$$
\begin{aligned}
& R_{t}(t)=R_{t, 0}+\int_{0}^{t} v(u) d u \\
& R_{r n}(t)=R_{r n, 0}+\int_{0}^{t} v(u) d u
\end{aligned}
$$

and the target echo delay becomes

$$
\tau_{n}(t)=\tau_{n}(0)+\frac{2}{c} \int_{0}^{t} v(u) d u
$$

A possible solution is to approximate the radial velocity with a Taylor series expansion as

$$
v(t)=v(0)+v^{\prime}(0) t+v^{\prime \prime}(0) \frac{t^{2}}{2}+\ldots+v^{(n)}(0) \frac{t^{n}}{n !}
$$

In this case, the backscattered signal under the narrowband approximation is

$$
\begin{aligned}
s_{n}(t) & =\tilde{b} \tilde{p}\left(t-\tau_{n}(0)\right) e^{-j 2 \pi f_{0} \tau_{n}(0)} e^{-j 2 \pi f_{0} \frac{2}{c} v(0) t} \\
& \times e^{-j 2 \pi f_{0} \frac{v^{\prime}(0) t^{2}}{c}} \times \ldots \times e^{-j 2 \pi f_{0} \frac{2 v^{(n)}(0) t^{n+1}}{c(n+1) !}}
\end{aligned}
$$

Because of the phase high order terms, it is not possible to achieve full integration with a typical Fourier Transform. These terms induce a temporal steering vector mismatch which against standard Doppler processing result in a loss in integration and hence a loss in detection performance. To solve this issue, techniques to detect and estimate signals with a polynomial phase may be applied as those developed in [4] [5].

It can be shown that the phase history of a target moving horizontally and perpendicularly to the radar boresight is quadratic. In this case a simple compensation technique can be implemented to achieve the same SNR as for the case with constant radial velocity .

\section{Simulation Results}

A set of simulations was carried out to study the integration efficiency of a staring radar of the type of the 3D Aveillant Holographic radar for ideal and partially-correlated point targets moving with both constant and non-constant radial velocity. The transmitting antenna was simulated as an isotropic element with phase centre located in the origins of a Cartesian reference system, whilst the receiver was implemented as a $4 \times 16$ receiver array of ideal omni-directional elements spaced of $\frac{\lambda}{2}$. For all simulations, the distance between the target and the radar was much greater than the size of the receiving array. The radar transmit signal was a linear chirp

$$
p(t)=e^{j \pi \gamma t^{2}} \operatorname{rect}\left[\frac{t}{\tau_{c}}\right]
$$

where

$$
\operatorname{rect}\left[\frac{t}{\tau_{c}}\right]=\left\{\begin{array}{rr}
1 & -\frac{\tau_{c}}{2}<t<\frac{\tau_{c}}{2} \\
0 & \text { elsewhere }
\end{array}\right.
$$

with chirp rate $\gamma=\frac{B_{c}}{\tau_{c}}$, pulse width $\tau_{c}$ and bandwidth $B_{c}$. The target was modelled as ideal point target with a 


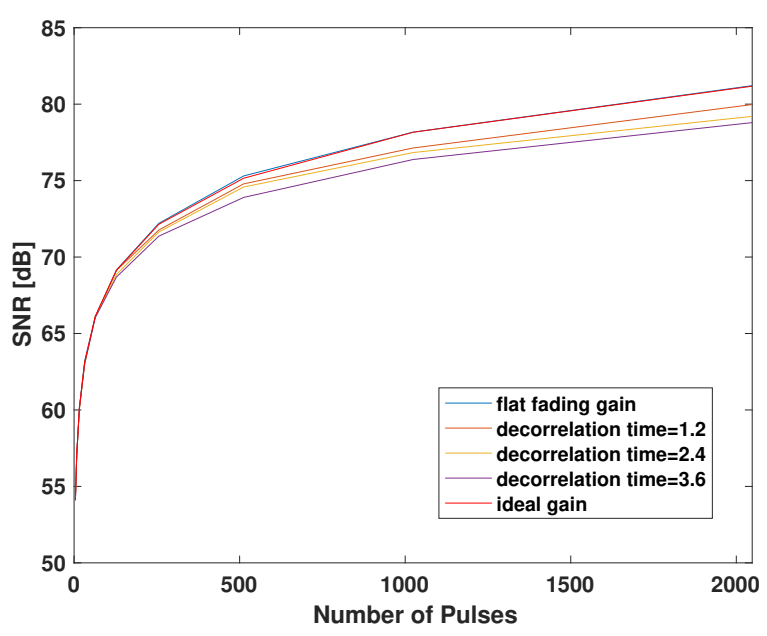

Fig. 1. Integration efficiency for a stationary target.

complex Gaussian response $\tilde{b}$ de-correlating from pulse to pulse exponentially as

$$
\left.E\left\{\tilde{b}^{*}(k+1) \tilde{b}^{(} k\right)\right\}=\sigma^{2} e^{-\beta|k| T}
$$

Table III contains the main parameters of the simulations. The

\begin{tabular}{|r|c|c|}
\hline Signal to Noise Ratio (1 pulse) & SNR & $30 \mathrm{~dB}$ \\
\hline Carrier Frequency & $f_{0}$ & $1.2 \mathrm{GHz}$ \\
\hline Pulse Repetition Frequency & PRF=1/PRI & $7.2 \mathrm{kHz}$ \\
\hline Pulse width & $\tau_{c}$ & $30 \mu \mathrm{s}$ \\
\hline Chirp Bandwidth & $B_{c}$ & $3 \mathrm{MHz}$ \\
\hline Sampling Frequency & $f_{s}$ & $45 \mathrm{MHz}$ \\
\hline Range & $R_{n, 0}$ & $1500 \mathrm{~m}$ \\
\hline Target Velocity & $V_{r}$ & $50 \mathrm{~m} / \mathrm{s}$ \\
\hline TABLE &
\end{tabular}

Simulations Parameters.

processing consisted of the following steps:

- simulating the transmitted and received signals at each element;

- modelling the noise as a complex circular white Gaussian random process with Power Spectral Density (PSD) to achieve the desired single element SNR (30 dB);

- modelling the target as a fluctuating target with response $\tilde{b}$

- compressing the received signal with the matched filter;

- implementing the digital beam forming;

- computing the discrete Fourier transform (DFT) of the slow-time data sequence;

- averaging 100 Monte Carlo simulations;

Fig.1 shows the SNR after integration versus the number of transmitted pulses $N_{p}$ and for different values of the parameter $\beta^{*}=\beta T$ for a stationary target. As expected the SNR for 1 pulse is the sum between the SNR at each element $(30 \mathrm{~dB})$ plus the beamforming gain $\left(10 \log _{10}(M \times N)=18 \mathrm{~dB}\right)$. The ideal gain and the average gain relative to $\beta^{*}=0$ (completely correlated target) equals $10 \log _{10} N_{P}$ leading to the highest SNR of $81 \mathrm{~dB}$ for 2048 pulses. As expected, the higher $\beta^{*}$

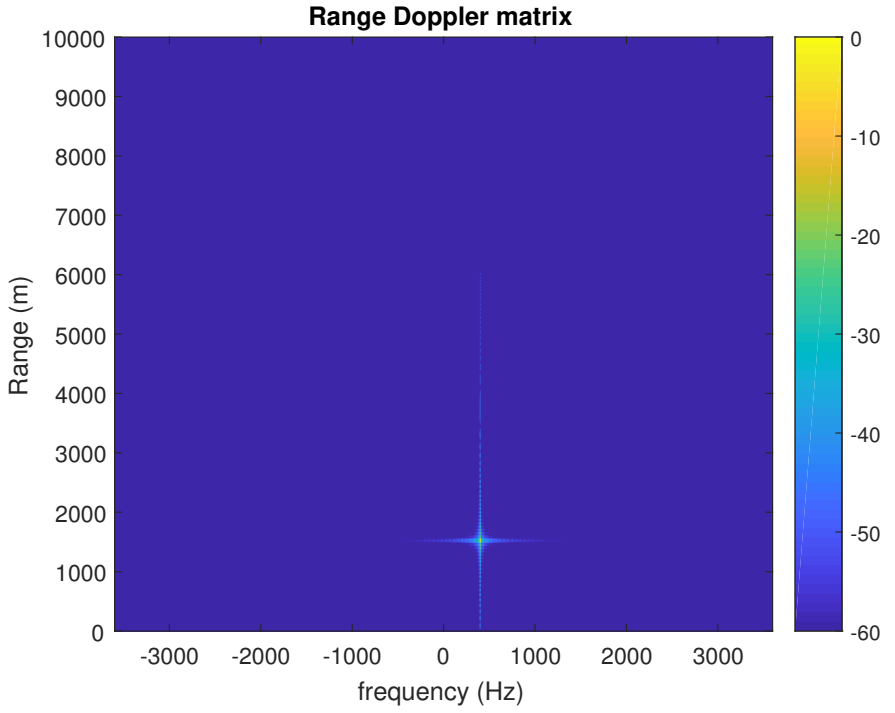

Fig. 2. Range Doppler map for a target moving with constant radial velocity.

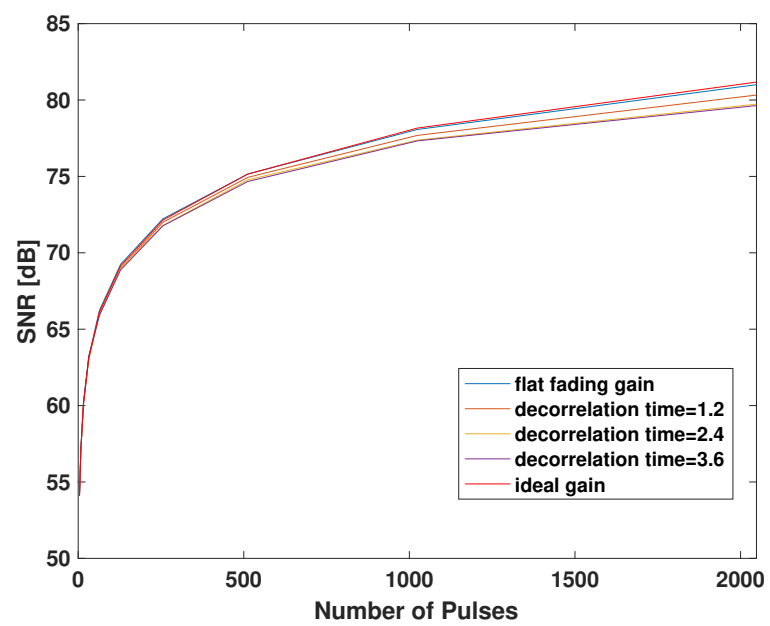

Fig. 3. Integration efficiency for a target moving with constant radial velocity after Fourier processing.

the faster the target decorrelates and the lower the gain.

Fig. 2 shows the range Doppler map for a target moving with a constant velocity of $50 \mathrm{~m} / \mathrm{s}$ obtained by integrating $N_{p}=2048$ pulses and Fig. 3 the values of the SNR versus the number of transmitted pulses $N_{p}$. Results are relative to a case when the normalised Doppler frequency exactly matched a DFT frequency bin, that is no straddle loss was present. As expected, in this case, range Doppler processing provides a perfect compensation of a constant Doppler frequency shift and after Fourier processing the results are equivalent to those of the static target. The range Doppler map relative to a target moving perpendicularly to and crossing the antenna boresight is shown in Fig. 4. In this case, the target radial velocity varies with time and the target Doppler shift spans a bandwidth consisting of positive and negative frequencies that cannot be compensated with standard Fourier Processing. 


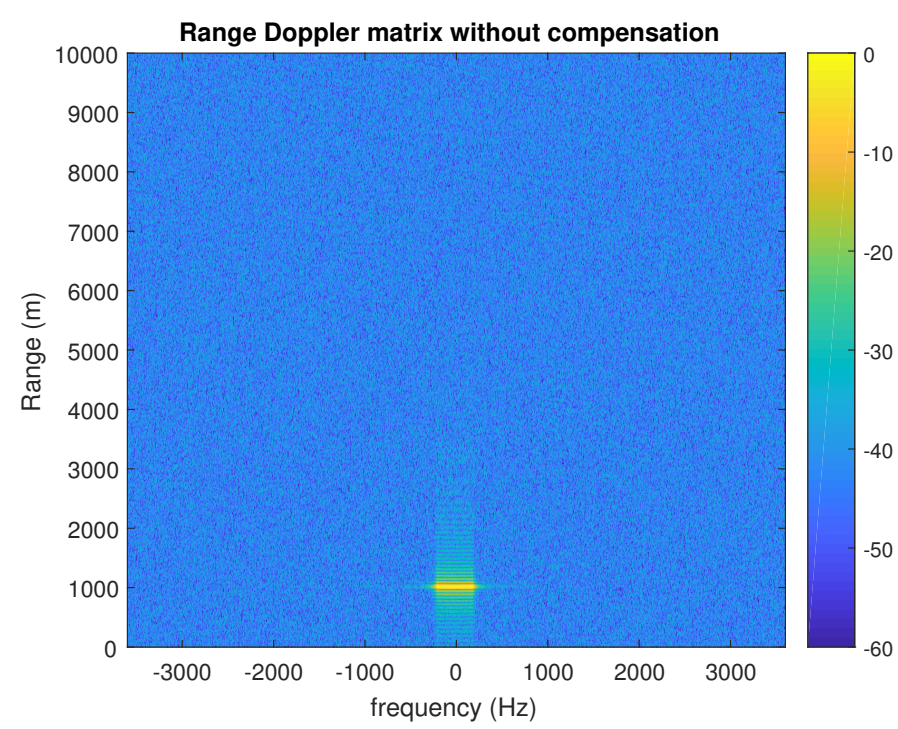

Fig. 4. Range-Doppler map before compensation for a target moving perpendicularly to LOS.

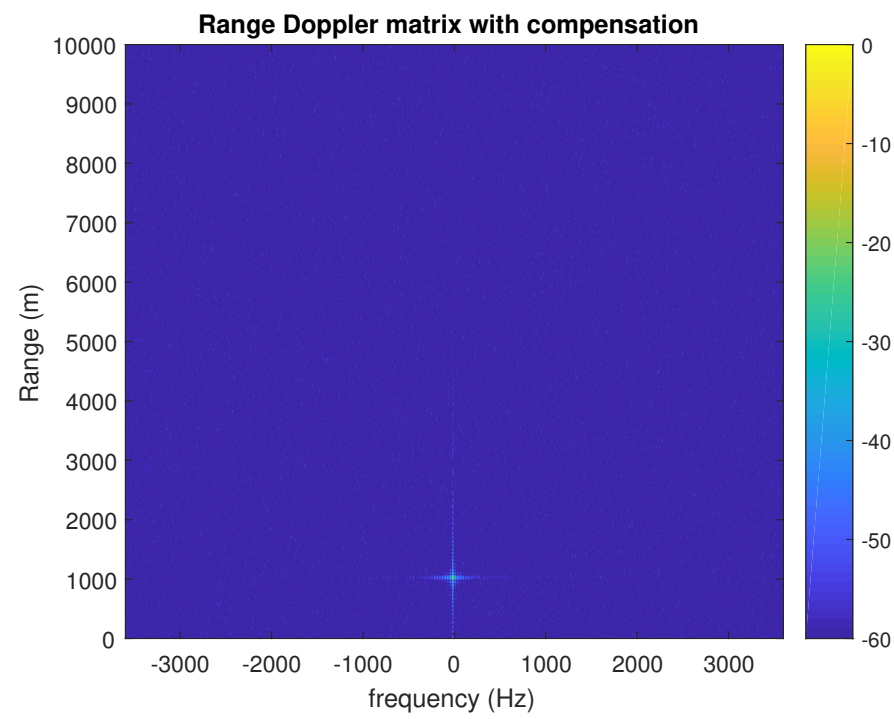

Fig. 5. Range-Doppler map after compensation for a target moving perpendicularly to LOS.

Results show a loss of approximately $35 \mathrm{~dB}$. Compensating the quadratic phase term produces the results in Fig. 5 for which the maximum SNR is retrieved.

\section{CONCLUSiOn AND Future Work}

This paper presents results to investigate the integration gain efficiency of staring radars that provide a significantly longer integration time than conventional rotating radars. Results are presented for the case of a target moving with constant radial velocity and for a target moving with non-constant radial velocity to show that, when the target Doppler shift varies as function of time, classical Fourier based techniques fail to provide optimal integration. The case of a target crossing boresight is studied to demonstrate that compensation techniques can be potentially employed to maximise SNR. Results are also shown for a partially-correlated target to emphasise the role played by the target response coherency and to show that, despite any possible compensation of phase terms deriving from the target trajectory, integration losses will occur also because the target response decorrelates.

Future work will focus on a detailed study of these technical challenges through the analysis of real data. Research will look at adapting and applying signal processing techniques, such as track before detect [6] and focus before detect [7] [8], to staring radars and at developing new solutions that can provide a complete exploitation of the staring radar dwell time, with a consequent improvement in SNR, independently from the target trajectory across the radar beam.

\section{ACKNOWLEDGMENT}

The authors would like to express their gratitude to Professor Antonio De Maio for his invaluable comments and suggestions on the paper.

\section{REFERENCES}

[1] M. Jahangir, C. J. Baker, and G. A. Oswald, "Doppler characteristics of micro-drones with 1-band multibeam staring radar," in Radar Conference (RadarConf), 2017 IEEE. IEEE, 2017, pp. 1052-1057.

[2] T. Quilter and M. Jahangir, "Performance of a 3d non-scanning array to detect targets in the presence of wind turbinesa step change for air traffic control," in Digital Communications-Enhanced Surveillance of Aircraft and Vehicles (TIWDC/ESAV), 2014 Tyrrhenian International Workshop on. IEEE, 2014, pp. 116-121.

[3] H. L. Van Trees, Detection, estimation and modulation theory, Part III. Radar-Sonar signal processing and Gaussian signals in noise. Wiley, 2001.

[4] S. Peleg and B. Friedlander, "The discrete polynomial-phase transform," IEEE Transactions on Signal Processing, vol. 43, no. 8, pp. 1901-1914, Aug 1995.

[5] S. Golden and B. Friedlander, "Estimation and statistical analysis of exponential polynomial signals," IEEE Transactions on Signal Processing, vol. 46, no. 11, pp. 3127-3130, Nov 1998.

[6] S. Buzzi, M. Lops, and L. Venturino, "Track-before-detect procedures for early detection of moving target from airborne radars," IEEE Transactions on Aerospace and Electronic Systems, vol. 41, no. 3, pp. 937-954, July 2005.

[7] J. Xu, Y. N. Peng, X. G. Xia, and A. Farina, "Focus-before-detection radar signal processing: part 1; challenges and methods," IEEE Aerospace and Electronic Systems Magazine, vol. 32, no. 9, pp. 48-59, September 2017.

[8] J. Xu, Y. N. Peng, X. G. Xia, T. Long, E. K. Mao, and A. Farina, "Focusbefore-detection radar signal processing: part 2;recent developments," IEEE Aerospace and Electronic Systems Magazine, vol. 33, no. 1, pp. 34-49, January 2018. 